\title{
Study on Measuring Method of Fiber Fusion Fitting in Substation
}

\author{
Xiaoxia Wu \\ School of North China Electric Power University, Baoding 071000, China. \\ 1278685811@qq.com
}

\begin{abstract}
Keywords: A/B/M Statistical Method, Data Processing, Optical Fiber Welding Quota Determination.
\end{abstract}
\begin{abstract}
Based on the characteristics of small sample and poor data of fiber splicing engineering, this paper analyzes and applies the knowledge of mathematical statistics, and puts forward a method of using T distribution theory, data leveling method to deal with data and make it stable. Furthermore, i developed a / b / m statistical method to determine the standard level of artificial, material, mechanical consumption. The amount of fiber consumption is determined by averaging the amount of material consumption. At the same time, this paper also proposed a measuring idea for seldom used fiber welding quota.
\end{abstract}

\section{Introduction}

With the rapid development of smart grid, fiber will be a lot of applications in the construction of the grid. However, there is no uniform pricing standard on the market at present. In order to unify the pricing standard of optical fiber welding, it is necessary to explore the scientific measurement method to calculate the cost of fiber welding to improve the pricing and charging of optical fiber, improve the cost management level of fiber welding project, complete and improve the existing quota system as well as its transparency, and to better serve the grid construction.

\section{Raw Data Acquisition}

Before calculating the quota, one is required to get the relevant data first. The data of fiber welding quota can be divided into three facets: labor, materials and machinery. Time measurement method can be used to measure the time labors spent, materials and mechanical consumption are able to determine by field statistics method.

\section{Data Processing}

The main purpose of the quota determination is to determine the level of consumption that can be used to reflect the majority of $(85 \%)$ units for this task according to existing engineering data, and for future cost estimates for similar projects.

However, due to the different geography of different projects, climate conditions, economic development level, the level of construction technology and other differences, the collection of engineering data is often uneven levels, and they may even differ by a few times. The direct use of these raw data for quantitative estimates is bound to result in a significant difference between the results and the actual average level, so it is necessary to carry out data processing. Through the data processing will identify and remove the abnormal value, then use scientific methods to normalize the data to meet the requirements of fiber welding consumption measurement.

In view of that fiber splicing is multi-step, data processing can take following five steps.

Step 1: Converts the data in the form of data collection to the percentage of total work time.

Fiber splicing can be divided into more than a dozen processes, the time spent on each process for different projects differs from each other, so that the total time gotten directly from the sum of all process will lead to greater differences. The average level of welding work may differ significantly from the actual results. In order to reduce the volatility between the data and accurately determine the average level of consumption of the fiber splice work, i decided to measure the average working hours 
per work by its percentage of the total working hours. That is, the percentage of each process of the total work time is calculated first. And then through the horizontal comparison the same process of a few works to determine the percentage of each process should be the percentage of the total hours of work.

Step 2: Removes the outliers according to the T distribution (Figure 1).

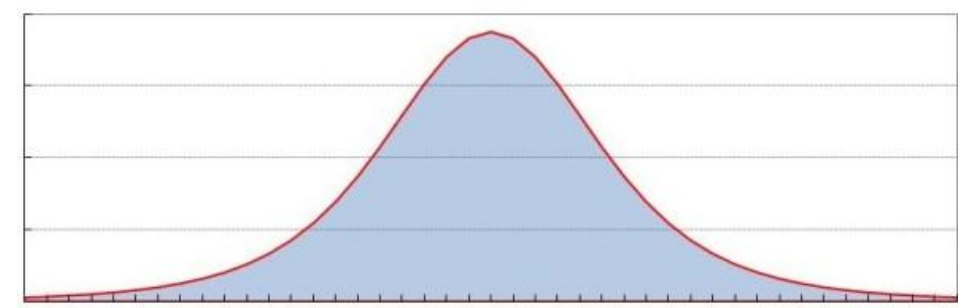

Fig.1 T distribution graph

The T distribution is often used to estimate the mean of the population of the normal distribution. In the project, the average level is defined as the level that $85 \%$ of the construction enterprises can achieve, according to which the reliability of fiber welding work can be set at $85 \%$. And then use the MATLAB program to obtain $T$ distribution results. And the data not in the confidence interval is removed to eliminate the effect of the outliers.

Step 3: Regulate the fit to determine the average of the percentages.

The linear regression of the percentages of each process is fitted by the MATLAB program. The average percentage of per process of the total work can be accurately determined according to the fitting curve and the fitting equation.

Step 4: Exception handling.

First determine an average level as the reference value (i.e., the average percentage value obtained in the third step above), multiply the reference value by 1.2 as the upper limit for making this group of data stable; 0.8 be able to keep this group of data to be stable. If the original data is less than the lower limit, then take the reference value of 0.8 times as the adjusted value; if the original data happens to fall between the lower limit and the upper limit, then the original data is not adjusted, which itself is valid; if the original data is greater than the upper limit, take the upper limit to replace the original value.

That is: set the reference value ${ }_{x}^{-}$, the original data is $x_{i}(0<\mathrm{i}<18)$, there are:

$$
\begin{cases}x_{i} \prec \bar{x} *(1+20 \%), & x_{i}=\bar{x} *(1-20 \%) \\ \bar{x} *(1-20 \%) \prec x_{i} \prec \bar{x} *(1+20 \%), & x_{i}=\bar{x} *(1+20 \%) \\ x_{i}>\bar{x} *(1+20 \%), & x_{i}=\bar{x} *(1+20 \%)\end{cases}
$$

After the above processing, you can achieve the data sequence which tends to be stable and access to valid data of consumption and cost of fiber welding.

Step 5: Use the percentage of each project in each of the projects identified above, multiply the original total hours of each project, then it is the result of the data processing. In this way, we adjust the unreasonable data in the raw data to facilitate the further analysis of the application of the data.

\section{Determination model}

Based on the data collected from the quota have the characteristics of poor data and few samples, $i$ construct an innovative and scientific method, i.e., the mathematical Statistics model of probability theory, it is named by a / b / m statistical method, to calculate the consumption that reflects the normal construction and management level.

The principle of $\mathrm{a} / \mathrm{b} / \mathrm{m}$ statistical method is to arrange a set of data in the order of size and find the arithmetic mean of the set of data. That is the general value of $m$, which indicates the average level represented by each project for the same fiber splicing process. According to the principle of the 
quadratic averaging method, the calculated values of the values less than the normal value $m$ are averaged on the set of data for $x_{\min }$, and the values greater than $\mathrm{m}$ in the set of data are averaged for $x_{\max }$. For the calculation of consumption, the greater the data means the greater the consumption and the lower level of construction. On the other hand, the smaller the value, the smaller the consumption, the higher the level of construction. Therefore, the advanced value $a$ is the arithmetic mean of $x_{\min }$ and the general value, which represents the average level of the advanced level of the construction technology. Similarly, the conservative value $b$ is arithmetic mean of $x_{\max }$ and the general value indicating the average level of the optical fiber welding is slightly lower in the construction skill level.

They can be show as follows:

$$
\mathrm{a}=\frac{\overline{\mathrm{x}_{\min }}+\mathrm{m}}{2}
$$

According to the statistical principle, a set of random data is subject to a normal distribution, as shown in figure 2.

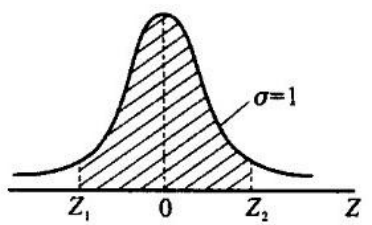

Fig. 2 Normal distribution graph

According to the graph of the $\mathrm{T}$ distribution, the smaller: the general value: the larger is close to

1: 4: 1. Then according to the principle of importance, the proportion of small means sub-important, accounting for a large proportion means more important. Thus the weight of the advanced value and the conservative value are taken as 1, the weighted average of the general value is 4. The weighted average is the final average level, and the average level thus determined is based on the distribution of the data and is more representative of the average than the average method.

Then, based on the mean distribution of the normal distribution formula, you can determine the average level of construction quota t:

$\mathrm{t}=\bar{t}-\lambda \sigma$

Where: $\mathrm{t}$-- the final determination of the construction quota level;

$\bar{t}$--weighted average;

$\sigma$-- Standard deviation;

$\lambda$-- Standard deviation coefficient, look-up the table.

In summary, the steps used for the determination of fiber splice quota is: 1)Using a quadratic average method obtains a, b, m value; (2)Apply a, b, m value have obtained into a / b / $\mathrm{m}$ statistical method to determine weighted average; (3) Calculate the standard deviation, the standard deviation coefficient and put them into normal distribution mean formula to decide the level of consumption.

\section{Empirical}

Assuming that the data of nine available 12-core fiber splicing projects are collected. As the same method, this paper only take the first process as an example to illustrate the specific operation (Table 1).

According to the normal distribution, the construction quota $t$ with the average advanced level is deduced. Take the first procedure as an example:

$\mathrm{t}_{1}=\bar{t}-\lambda \sigma=0.54-1.037 \times 0.01=0.53$

The same can calculate the consumption of the remaining processes, the total can be 12 core fiber welding consumption standards. 
Table 1. Major Data Processing

\begin{tabular}{|c|c|c|c|r|r|r|r|r|c|}
\hline Steps Project & 1 & 2 & 3 & 4 & 5 & 6 & 7 & 8 & 9 \\
\hline $\begin{array}{c}\text { Raw Data } \\
\text { (working hours) }\end{array}$ & 0.09 & 0.60 & 0.65 & 0.54 & 0.48 & 0.37 & 0.73 & 0.44 & 0.49 \\
\hline $\begin{array}{c}\text { Percentage after } \\
\text { Conversion }\end{array}$ & $0.69 \%$ & $5.82 \%$ & $5.62 \%$ & $5.50 \%$ & $5.08 \%$ & $5.25 \%$ & $5.44 \%$ & $5.27 \%$ & $6.26 \%$ \\
\hline $\begin{array}{c}\text { Data Removed } \\
\text { (working } \\
\text { hours) }\end{array}$ & 0.007 & 0.058 & 0.056 & 0.055 & 0.051 & 0.053 & 0.054 & 0.052 & 0.063 \\
\hline New Percentage & $4.33 \%$ & $5.82 \%$ & $5.62 \%$ & $5.50 \%$ & $5.08 \%$ & $5.25 \%$ & $5.44 \%$ & $5.27 \%$ & $6.26 \%$ \\
\hline $\begin{array}{c}\text { Dealing Results } \\
\text { (working hours) }\end{array}$ & 0.54 & 0.60 & 0.65 & 0.54 & 0.48 & 0.37 & 0.73 & 0.44 & 0.49 \\
\hline
\end{tabular}

Note: The yellow shaded data in the above table indicates abnormal data that needs to be removed.

\section{Summary}

This paper combines with the characteristics of actual situation and models a set of fiber welding consumption measurement method. The method is simple and feasible, which lays a good foundation for perfecting the establishment of fiber splice quota and provides a scientific basis for the bidding of construction enterprises. It also helps to improve the management level and regulate the market behavior as well as strengthens the market competition.

\section{References}

[1]. Juan Du. Application of optical fiber communication in power dispatching automation [J]. Science and Technology Innovation and Application. Vol. 23 (2014) No. 11, p. 73.

[2]. Information on: http://baike.so.com.

[3]. Xiaozhou Wang. Fuzzy prediction method of artificial and main material consumption in construction engineering. Journal of Wuyi University. Vol. 15 (2001) No. 2, p. 47-50.

[4]. Hongying Fang. Research on Data Preprocessing in Data Mining. (Master Degree. Southwest University. China. 2009) p. 4-26. 